\section{G109 OBESITY IN CHILDREN WITH AUTISM SPECTRUM DISORDER AUDIT}

doi:10.1136/archdischild-2013-304107.121

'EK Grylls, ${ }^{2 M}$ Coxhead, ${ }^{2 P}$ Dehiwelage, 'S Thomas. 'The Public Health Department, Northeast Essex PCT, Essex, UK; ${ }^{2}$ The Community Paediatrics Department, Colchester Hospital University Foundation Trust, Essex, UK

Aims To audit whether children in the region studied diagnosed with autism spectrum disorder (ASD) are being measured in accordance with the national child measurement programme. To define the prevalence of overweight/obesity in these children and evaluate how well they were being managed, against the NICE guidelines and the healthy child programme.

Methods Medical notes were analysed from a convenience sample of 77 children with ASD from both special (59) and mainstream (18) schools in the region studied.

Results Eighty five per cent and $82 \%$ of the eligible children were being measured in reception and year 6 , respectively. Forty seven per cent of the children were overweight/obese, of which a higher proportion were from special schools (53\%) than mainstream schools $(28 \%)$. A concern had been raised in only $51 \%$ of these children. BMI was calculated in only $5 \%$ of all children. Where a concern was raised interventions including discussion with parents $(95 \%)$, advice on diet/exercise (77\%), regular weighing $(91 \%)$ and referral to the dietician $(86 \%)$ were done in most cases. However, only $18 \%$ were referred to the local healthy lifestyle programme and there were concerns raised by the community paediatricians and the lead coordinator of the local healthy lifestyle programme that this service is not suitable for all children with ASD. Of the obese children, $62 \%$ were investigated for comorbidities.

Conclusion Children with ASD were being measured in accordance with the national child measurement programme. A high proportion were found to be overweight/obese. This audit highlighted significant deviation from national guidelines in the detection and management of the overweight/obese children. The low proportion of cases in which BMI was calculated may have contributed to almost half of the children not being identified as overweight/obese. The need for a local healthy lifestyle programme tailored to children with ASD has been highlighted to the public health team locally. This study has informed local practise and service requirement. Similar studies may be beneficial elsewhere.

\section{G110(P) FACIAL NERVE PALSY IN MOEBIUS SEQUENCE - CAN PRACTISE MAKE PERFECT?}

doi:10.1136/archdischild-2013-304107.122

${ }^{1.3} \mathrm{~N}$ Gerson-Sofer, 'L Sanalla-Shehadeh, ${ }^{2} \mathrm{D}$ Savitzki. 'Child Development Centre, Western Galilee Hospital, Naharia, Israel; ${ }^{2}$ Child Neurology Unit, Western Galilee Hospital, Naharia,Israel; ${ }^{3}$ Medical School, Bar Ilan University, Zefat, Israel

Moebius Sequence or Syndrome (MBS) is an extremely rare ( $\sim 0.002 \%$ of births) congenital, nonprogressive facial weakness with limited abduction of one or both eyes. Additional features can include deafness and other cranial nerve dysfunction, motor, orofacial, musculoskeletal, neurodevelopmental, and socialbehavioural problems. Various aetiologies are implicated, including familial 13q12.2-q13 deletion, intrauterine teratogen exposure (misoprostol, cocaine, thalidomide), vascular disruption and congenital myopathy, all potentially affecting development of the sixth and seventh nerve nuclei. Lack of facial expression (inability to smile), difficulties in acquiring effective oral control (drooling), and unintelligible speech due to extremely limited range of vowels and phonemes contribute to significant social and communication impairments. Inability to smile may compromise parental emotional bonding.
Aims (1) to document response to intensive speech/language intervention with progress in ability to formulate speech sounds in a young child with MBS;

(2) to summarise current approaches, including surgical, for rehabilitation of facial animation and speech, and limitations thereof.

Methods A case presentation with serial videoclips depicting speech therapy and speech production in a toddler with MBS over 18 months of intervention.

Results Significant progress was made in production of speech sounds in a toddler with MBS using speech-language techniques developed for children with Childhood Speech Apraxia. Intelligibility remains limited, although improved. Current surgical options for muscle or nerve transfer are considered for improving facial animation and speech.

Conclusion MBS is extremely rare, and causes significant social and communication difficulties. A child with normal cognitive abilities and language comprehension born with bilateral congenital abducens and facial nerve palsy was given intensive oral stimulation and speech-language training using techniques developed for Childhood Speech Apraxia. Ability to formulate certain vowels and phonemes was gained. However acquisition of the whole range of tongue and mouth movements and speech sounds is probably restricted. Intensive therapy can improve speech in congenital facial nerve palsy, but incorporation of surgical muscle or nerve transfers and hopefully future technologies will probably be required to normalise facial animation and formulation of speech sounds. Therefore, in the current state of art, practise cannot make perfect.

\section{G111(P) THE ROLE OF MODAFINIL IN IMPROVING MOTOR FUNCTION IN CEREBRAL PALSY: A CASE STUDY}

doi:10.1136/archdischild-2013-304107.123

${ }^{1} \mathrm{M}$ Crouchman, ${ }^{2} \mathrm{H}$ Greenberg, ${ }^{3} \mathrm{R}$ Hughes. 'Department of Neuropaediatrics, The Portland Hospital for Women and Children, London, UK; ${ }^{2}$ Department of Anaesthetics, Northwick Park Hospital, London, UK; ${ }^{3}$ Neuropaediatric Physiotherapy, Private Practice, London, UK

Background An American study showing improved Gross Motor Function (GMF) in cerebral palsy (CP) attributed this to a direct action on spasticity of modafinil, a drug used to treat Attention Deficit/Hyperactivity Disorder (ADHD). There is no other evidence that modafinil reduces spasticity.

Purpose To clarify modafinil's mode of action.

Methods Our subject was a 9 year old girl with spastic diplegia, seen previously by a number of paediatricians. Following clinical assessment the GMF score and the modified Connor score for $\mathrm{ADHD}$ were recorded. These were repeated after one month's treatment with modafinil.

Results Her initial Connor score was $21 / 30$ (very high), and fell to $5 / 30$. Her GMF rose from 28 (25\% of norm) to 139 (49.8\%). A detailed history from her mother and clinical examination suggested ADHD. During treatment she had a growth spurt, and after one month her spasticity had increased and her gait deteriorated. However, she had better control of her walking aid, she achieved alternation of her feet on stair climbing and improved ability to handle cutlery, she was able to undress herself for the first time, her stamina and tolerance of physiotherapy improved and her speech and writing became clearer. There were also marked improvements in cognitive and social functioning; she gained 2 levels in reading and read independently for the first time, she was less emotionally labile, she slept better and became more appropriate in behaviour and interactions. She expressed pleasure in these gains.

Conclusions Motor improvement occurred despite increased spasticity. It was associated with reduction in both distractibility and excessive movements, both symptomatic of $\mathrm{ADHD}$, and it is probable that treatment for this condition improved her motor function. 